\title{
Pressure spectra from single-snapshot tomographic PIV
}

\author{
Jan F. G. Schneiders ${ }^{1} \mathbb{D} \cdot$ Francesco Avallone $^{1} \cdot$ Stefan Pröbsting ${ }^{1} \cdot$ Daniele Ragni $^{1} \cdot$ Fulvio Scarano $^{1}$
}

Received: 24 May 2017 / Revised: 8 January 2018 / Accepted: 13 January 2018 / Published online: 26 February 2018

(c) The Author(s) 2018. This article is an open access publication

\begin{abstract}
The power spectral density and coherence of temporal pressure fluctuations are obtained from low-repetition-rate tomographic PIV measurements. This is achieved by extension of recent single-snapshot pressure evaluation techniques based upon the Taylor's hypothesis (TH) of frozen turbulence and vortex-in-cell (VIC) simulation. Finite time marching of the measured instantaneous velocity fields is performed using TH and VIC. Pressure is calculated from the resulting velocity time series. Because of the theoretical limitations, the finite time marching can be performed until the measured flow structures are convected out of the measurement volume. This provides a lower limit of resolvable frequency range. An upper limit is given by the spatial resolution of the measurements. Finite time-marching approaches are applied to low-repetition-rate tomographic PIV data of the flow past a straight trailing edge at $10 \mathrm{~m} / \mathrm{s}$. Reference results of the power spectral density and coherence are obtained from surface pressure transducers. In addition, the results are compared to state-of-the-art experimental data obtained from time-resolved tomographic PIV performed at $10 \mathrm{kHz}$. The time-resolved approach suffers from low spatial resolution and limited maximum acquisition frequency because of hardware limitations. Additionally, these approaches strongly depend upon the time kernel length chosen for pressure evaluation. On the other hand, the finite timemarching approaches make use of low-repetition-rate tomographic PIV measurements that offer higher spatial resolution. Consequently, increased accuracy of the power spectral density and coherence of pressure fluctuations are obtained in the high-frequency range, in comparison to the time-resolved measurements. The approaches based on TH and VIC are found to perform similarly in the high-frequency range. At lower frequencies, TH is found to underestimate coherence and intensity of the pressure fluctuations in comparison to time-resolved PIV and the microphone reference data. The VIC-based approach, on the other hand, returns results on the order of the reference.
\end{abstract}

\section{Introduction}

Measurements of the unsteady pressure fluctuations, their time spectrum and their coherence are relevant in aerodynamics, aero-elasticity and aero-acoustics, since they provide information for estimation of the unsteady loads and noise sources. In the past two decades, pressure from particle image velocimetry (PIV) has received significant attention from the scientific community. It is an alternative to wind tunnel model instrumentation with pressure transducers, or pressure-sensitive paint (PSP; Bitter et al. 2012), which has

Electronic supplementary material The online version of this article (https://doi.org/10.1007/s00348-018-2507-2) contains supplementary material, which is available to authorized users.

Jan F. G. Schneiders

j.f.g.schneiders@tudelft.nl

1 Department of Aerospace Engineering, TU Delft, Delft, The Netherlands limited sensitivity in the low-speed regime. Many research groups have focused their efforts on all parts of the pressurefrom-PIV measurement chain, as summarized in Table 1. Starting from the measurement techniques (Table 1a), researchers have considered time-resolved high-repetitionrate PIV measurements, but also multi-pulse and dual-pulse (resp. single-snapshot) approaches. The measurement data is subsequently processed using either a cross-correlation or Lagrangian particle tracking (LPT) approach (Table 1b). All existing approaches obtain the pressure gradient from the velocity measurements by invoking the momentum equation. Depending on the measurement setup and data analysis procedure, many techniques to obtain the pressure gradient have been proposed (Table 1c). Finally, also the subsequent integration of the pressure gradient remains a topic of current research (Table 1d). In Table 1e salient comparative assessments that compare part of the above procedures are listed. Note that the majority of these works use volumetric measurement data, following recent research that shows this 
Table 1 Pressure-from-PIV research overview. The numbers in the first column indicate the topic of the work: (a) measurement setup, (b) data interpretation, (c) pressure gradient evaluation, (d) pressure calculation, and (e) comparative assessments. Salient and pioneering works related to pressure-from-PIV are cited for each topic

\begin{tabular}{ll}
\hline a & High-repetition-rate \\
& Multi-pulse \\
& Double-pulse (i.e., single-snapshot) \\
b & Cross-correlation (PIV) \\
& Lagrangian particle tracking (LPT) \\
c $\quad \begin{array}{l}\text { Eulerian } \\
\text { Pseudo-Lagrangian }\end{array}$ & Lagrangian (LPT only) \\
& Dense interpolation (LPT only) \\
& Modelling \\
Gradient integration & FFT integration \\
& Poisson solution (gridded data) \\
& Poisson solution (scattered data) \\
Comparative assessments
\end{tabular}

Baur and Köngeter (1999); Schröder et al. (2006); Kotsonis et al. (2011); Ghaemi et al. (2012); Joshi et al. (2012)

Liu and Katz (2006); Lynch and Scarano (2014); Novara et al. (2016)

Gurka et al. (1999); Hosokawa et al. (2003); de Kat and Ganapathisubramani (2012); Laskari et al. (2016); Schneiders et al. (2016)

Adrian and Westerweel (2011); Elsinga et al. (2006); Lynch and Scarano (2013); Jeon et al. (2014)

Novara and Scarano (2013); Schanz et al. (2016)

Baur and Köngeter (1999); Dong et al. (2001); Jensen and Pedersen (2004); Perret et al. (2006) Liu and Katz (2006); Pröbsting et al. (2013); Wang et al. (2017a)

Ferrari and Rossi (2008); Novara and Scarano (2013); Novara et al. (2016); Schanz et al. (2016) Gesemann et al. (2016); Schneiders and Scarano (2016)

de Kat and Ganapathisubramani (2012); Laskari et al. (2016); Schneiders et al. (2016)

Imaichi and Ohmi (1983); Baur and Köngeter (1999); Haigermoser (2009); Liu and Katz (2006)

Huhn et al. (2016); Wang et al. (2017b)

Fujisawa et al. (2005); de Kat and van Oudheusden (2012); Ragni et al. (2012); Tronchin et al. (2015)

Neeteson and Rival (2015); Neeteson et al. (2016)

Charonko et al. (2010); Violato et al. (2011); de Kat and van Oudheusden (2012); van Gent et al. (2017); McClure and Yarusevych (2017) is a requirement to accurately obtain the pressure gradient (Charonko et al. 2010; de Kat and van Oudheusden 2012; Ghaemi et al. 2012). It should be remarked that some works have considered pressure evaluation in compressible flows (e.g., van Oudheusden et al. 2007; Ragni et al. 2009), but the present discussion and work is limited to incompressible, isothermal flows and Newtonian fluids.

Despite the above developments, recent works have not been able to measure pressure spectra and coherence at high frequencies (de Kat and van Oudheusden 2012; Ghaemi et al. 2012; Pröbsting et al. 2015). Even though these studies used time-resolved tomographic PIV at frequencies up to $10 \mathrm{kHz}$, the pressure spectra were found to be dominated by measurement errors well before the Nyquist frequency. This is a direct consequence of the limited spatial resolution of available time-resolved pressurefrom-PIV techniques (see e.g. Lynch et al. 2014; Charonko et al. 2010; de Kat and van Oudheusden 2012; McClure and Yarusevych 2017). The largest resolvable frequency of pressure fluctuations that can be estimated depends on the smallest resolvable scale. The latter is approximately twice the interrogation volume (IV) size used for PIV cross-correlation analysis (Schrijer and Scarano 2008). Accordingly, the largest frequency of pressure fluctuations that can be measured is given by:

$f_{\max } \approx \frac{u_{\mathrm{c}, \mathrm{p}}}{2 \mathrm{IV}}$ where $u_{\mathrm{c}, \mathrm{p}}$ is the convective speed of the pressure fluctuations. For example, in case the turbulent boundary layer measurements by Pröbsting et al. (2015), a window size of $1 \mathrm{~mm}$ was achieved. This resulted in a maximum resolved frequency of approximately $3 \mathrm{kHz}$, despite the use of a timeresolved measurement system operating at $10 \mathrm{kHz}$.

The comparative assessment in van Gent et al. (2017) shows that a better result (i.e., higher spatial resolution) could be obtained using Lagrangian particle tracking (Table 1b). However, due to limited measurement rates, Lagrangian particle tracking (LPT) remains limited to relatively low-speed flows to allow accurate particle pairing and tracking. Multi-pulse approaches (Table 1a) overcome these measurement rate limitations. However, these techniques return uncorrelated velocity and pressure fields that do not directly allow for evaluation of the spectrum and moreover require relatively expensive setups with an extended number of imagers and lasers.

Avoiding the need for additional hardware, a similar result can be obtained using single-snapshot approaches, that have been introduced to approximate instantaneous pressure from standard low-repetition-rate tomographic PIV (de Kat and Ganapathisubramani 2012; Laskari et al. 2016; Schneiders et al. 2016). These techniques invoke modeling approaches to approximate the velocity material derivative that otherwise would be measured by time-resolved or multi-pulse approaches. The comparative assessment by van Gent et al. (2017) shows that single-snapshot approaches 
are less accurate than the time-resolved ones. This conclusion, however, does not take into account that singlesnapshot approaches can make use of a low-repetition-rate tomographic PIV system, which can benefit from increased measurement dynamic range due to the availability of larger format CCD cameras (see e.g. Hain et al. 2008; Schanz et al. 2012) and more powerful lasers with respect to the ones used for time-resolved measurements. This allows to employ a smaller interrogation volume size for cross-correlation analysis. However, the established single-snapshot approaches (Table 1a) return only uncorrelated pressure fields, and therefore, require an additional modeling assumption to obtain pressure spectra and coherence. Due to the increased spatial resolution with respect to a time-resolved approach, the result could, however, have higher accuracy.

To extract temporal pressure spectra and coherence from the velocity snapshots, a logical first step is to invoke Taylor's hypothesis of frozen turbulence. This was already done for single-snapshot pressure evaluation (de Kat and Ganapathisubramani 2012; Laskari et al. 2016). In addition, Scarano and Moore (2011) proposed a local advection-only approximation of the Navier-Stokes equations to approximate the temporal velocity fluctuations between correlated PIV measurement time instants, yielding an increased temporal resolution. This was called as time-supersampling. Schneiders et al. (2014) later generalized this technique by avoiding the linearization and use the incompressible vorticity transport equation as model for time-supersampling.

It should be remarked that the problem of supersampling measurement data has also been approached with more complex methods that take further into account the non-linear dynamics in the temporal evolution of the velocity field. In this respect, several methods have been proposed that use a variational approach to assimilate data from measurements and numerical simulations (Suzuki et al. 2009; Cuzol and Mémin 2009; Lemke and Sesterhenn 2016; Yegavian et al. 2015; Schneiders and Scarano 2016; Gesemann et al. 2016). Such variational approaches have proven to be successful in increasing the spatial resolution of three-dimensional time-resolved measurements (Schneiders and Scarano 2016; Gesemann et al. 2016; Schneiders et al. 2017). However, the literature shows no successful application of variational data assimilation to increase the temporal resolution of volumetric flow measurements.

Single-snapshot pressure evaluation techniques (de Kat and Ganapathisubramani 2012; Laskari et al. 2016; Schneiders et al. 2016) so far have not included a time-marching scheme for velocity and pressure, and therefore, do not allow for spectral information to be obtained when applied to lowrepetition-rate PIV data. Combination of the single-snapshot pressure evaluation methods and the abovementioned timesupersampling techniques could allow for evaluation of short time segments of the temporal pressure fluctuations from uncorrelated tomographic PIV measurement data. This is different from, for example, Schneiders et al. (2016), which only evaluates the instantaneous velocity material derivative from the approximated vorticity temporal derivative. The instantaneous velocity material derivative allows calculation of instantaneous pressure, but the scheme used by Schneiders et al. (2016) does not include time marching and no pressure time series is obtained when the technique is applied to instantaneous data.

The current work, instead, investigates the feasibility to obtain finite time segments of the pressure from low-repetition-rate volumetric velocity measurements using Taylor's hypothesis and a non-linear technique based on solution of the vorticity transport equation. The approaches are assessed for low-repetition-rate (less than $1 \mathrm{~Hz}$ ) tomographic PIV measurements in the turbulent boundary layer near the trailing edge of a flat plate. The proposed methods are used to obtain the pressure spectrum in the kilo-Hertz range. For validation, the results are compared to a reference spectrum obtained by surface pressure transducer. In addition, the methods are compared to state-of-the-art time-resolved tomographic PIV results.

\section{Finite time marching}

To obtain a finite time segment of the velocity and pressure temporal evolution from a single velocity field, information from the spatial domain can be used to increase temporal resolution. The measured velocity field (Fig. 2a, blue volume) is marched forward in time over a finite time segment. At time $t+\Delta T$ the volume has marched downstream over a distance of approximately $u_{\mathrm{c}, \mathrm{u}} \Delta T$ (Fig. $2 \mathrm{~b}$, orange volume), where $u_{\mathrm{c}, \mathrm{u}}$ is the convective speed of the velocity fluctuations. Accordingly, erosion of the measurement region starting from the inflow boundary is expected.

Finite time marching of the measured velocity field can be performed using the Taylor's hypothesis of frozen turbulence as follows (Scarano and Moore 2011):

$\boldsymbol{u}\left(\boldsymbol{x}, t_{0}+\Delta t\right)=\boldsymbol{u}\left(\boldsymbol{x}-\boldsymbol{u}_{c, u} \Delta t, t_{0}\right)$

where $u_{\mathrm{c}, \mathrm{u}}$ is the local convective speed of velocity. This speed is not generally known and needs to be selected. Laskari et al. (2016) compared several choices for determining the appropriate convective velocity for single-snapshot pressure evaluation in case of a turbulent boundary layer. It was concluded that the use of a streamwise mean of the velocity field yields most accurate results.

Alternatively, the work of Schneiders et al. (2014) provides a generalization of the above procedure to flows where the convective speed cannot be estimated, by performing a time-marching simulation of the incompressible vorticity 
transport equation using the vortex-in-cell technique (VIC, Christiansen 1973):

$\frac{\partial \boldsymbol{\omega}}{\partial t}=(\boldsymbol{\omega} \cdot \nabla) \boldsymbol{u}-(\boldsymbol{u} \cdot \nabla) \boldsymbol{\omega}+\nu \nabla^{2} \boldsymbol{\omega}$,

where in the present study the viscous term is included. Within the VIC framework, velocity is related to vorticity through:

$\nabla^{2} \boldsymbol{u}=-\nabla \times \omega$.

Boundary conditions for the time-marching simulation are obtained by finite-difference solution of the vorticity transport equation. Boundary values of velocity are set following the padding procedure used in Schneiders et al. (2016). The VIC technique in Schneiders et al. (2014) requires imposing boundary values of vorticity, which are obtained by finitedifference solution of the vorticity transport equation. The measurement volume is padded by a buffer region (gray area in Fig. 1), where the streamwise average velocity is initially prescribed. During the time-marching simulation, the information in the measurement volume region convects into this buffer region as illustrated in Fig. 1. The extent $L_{\mathrm{p}}$ of domain padding is determined by the integration time, $T$, and a typical velocity value representative of flow convection:

$L_{\mathrm{p}} \geqslant u_{\mathrm{c}, \mathrm{u}} T$.

Subsequently, the pressure temporal evolution in each time segment is obtained using an established pressurefrom-PIV approach that solves the Poisson equation for pressure (Table 1d). Boundary conditions on the pressure gradient (von Neumann type) are obtained from the momentum equation and are prescribed on all boundaries except one (i.e., top boundary in Fig. 1), where Dirichlet-type boundary conditions are assigned. The latter can be assigned as the free-stream mean pressure level, if part of the measurement volume extends up to the free stream, or it can be approximated from a model such as the Bernoulli equation or isentropic relations (Kurtulus et al. 2007; Ragni et al. 2009; de Kat and van Oudheusden 2012; among others).

The velocity material derivative, $\mathrm{D} u / \mathrm{D} t$, is calculated in Eulerian formulation (Table 1c).

$\frac{\mathrm{D} \boldsymbol{u}}{\mathrm{D} t}=\frac{\partial \boldsymbol{u}}{\partial t}+(\boldsymbol{u} \cdot \nabla) \boldsymbol{u}$,

where the temporal derivative is obtained using a secondorder central difference scheme from the velocity time series. The Eulerian formulation can be used because the methods allow for selection of a sufficiently small time step $\Delta t$ for time-marching to allow Eulerian derivative evaluation.

The statistical convergence of the pressure spectrum estimation is achieved by averaging the spectra obtained from time-segments from multiple uncorrelated tomographic PIV snapshots. This is analogous to Welch's power spectral density estimation procedure (Welch 1967). The short time segments require a windowing function. To avoid a discontinuity at the edges, a Hann window is used.

(b)

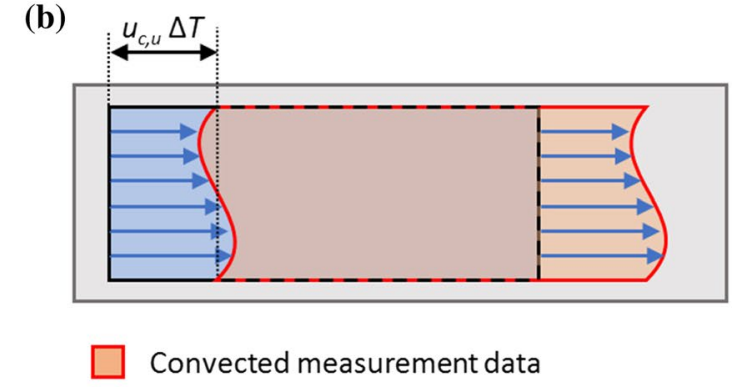

Convected measurement data (a)

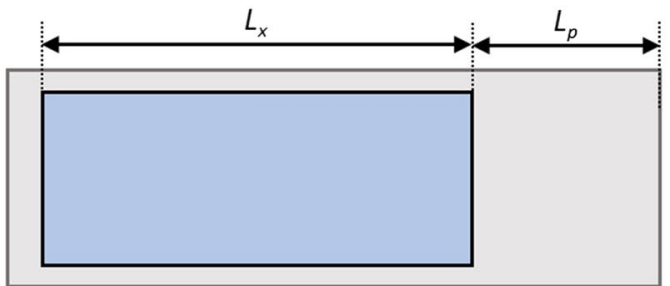

PIV data

Computational volume

Fig. 1 Schematic visualization of data erosion during a simulation over $\Delta T$. The left figure shows the PIV measurement data in the computational volume. The right figure shows data convected downstream after a time-marching VIC simulation over a time segment of $\Delta T$

Fig. 2 Measurement volume (green box) and microphone location (indicated in red). The time-averaged boundary layer profile at $x=-15 \mathrm{~mm}$ is indicated in orange






\section{Limitations}

\subsection{Dynamic frequency range}

The theoretical maximum duration of a time segment, $T_{\max }$, corresponds to the time when all the measured fluid parcels are convected out of the measurement volume. This can be estimated as the ratio of the streamwise measurement domain length, $L_{x}$, and a typical convective velocity in the flow, $u_{\mathrm{c}}$,

$T_{\max }=\frac{L_{x}}{u_{\mathrm{c}}}$.

Accordingly, the lowest resolvable frequency is

$f_{\min }=\frac{1}{T_{\max }}=\frac{u_{\mathrm{c}}}{L_{x}}$.

It should be remarked that in a shear flow, different parts of the volume are convected out of the measurement in different moments. In such a case, the maximum velocity is to be taken as characteristic velocity. Additionally, numerical instabilities or end effects may reduce the maximum time-segment time. As a consequence, the above equations serve only as an indication of the achievable $f_{\min }$.

The highest resolvable frequency is limited by the available spatial resolution. An estimate can be obtained considering the ratio of the smallest resolved length scale, $\lambda_{\min }$, and a typical convective velocity,

$f_{\text {max }} \approx \frac{u_{\mathrm{c}}}{\lambda_{\text {min }}}$.

Considering spatial cross-correlation for the analysis of the particle images, the smallest resolved length scale of velocity fluctuations is a function of the size of the interrogation volume, IV. According to Schrijer and Scarano (2008), a spatial modulation up to $50 \%$ of the velocity fluctuation is achieved when $\lambda=2 \mathrm{IV}$. Equation (3) can, therefore, be rewritten as:

$f_{\text {max }} \approx \frac{u_{\mathrm{c}}}{2 \mathrm{IV}}$.

In conclusion, the theoretical frequency dynamic range (DFR), given as the ratio of the highest to lowest resolvable frequency, reads as

$\mathrm{DFR}=\frac{L_{x}}{2 \mathrm{IV}}$.

Interestingly, the above expression corresponds to the dynamic spatial range, DSR, introduced by Adrian (1997) multiplied by a coefficient. The latter is a direct consequence of the fact that the proposed technique evaluates the pressure spectrum from measurement data distributed in the spatial domain only.

\subsection{Modeling limitations}

In addition to limitations on the achievable frequency range (Sect. 3.1), the theoretical limitations of single-snapshot pressure from PIV modeling approaches also hold for the present work (Schneiders et al. 2016). It was shown that only pressure fluctuations caused by measured flow structures are reconstructed. External effects caused by, for example, pulsating jets or a flapping wing are not predicted by the modeling approach, and therefore, the corresponding pressure fluctuations are not measured.

\section{Description of experiments}

The approaches discussed in Sect. 2 are applied to a tomographic PIV measurement at the trailing edge of a flat plate (Fig. 3) installed in a low-speed wind tunnel of $40 \times 40 \mathrm{~cm}^{2}$ cross-section operating at free-stream velocity of $10 \mathrm{~m} / \mathrm{s}$. The flat plate is $60 \mathrm{~cm}$ long, $40 \mathrm{~cm}$ wide and is $1 \mathrm{~cm}$ thick. The flat plate thickness decreases to $0.2 \mathrm{~mm}$ at the trailing edge with an angle of $2.4^{\circ}$ on both sides, which creates a mild pressure gradient. The boundary layer is tripped $10 \mathrm{~cm}$ downstream an elliptical leading edge using a $1 \mathrm{~cm}$ strip of 3D carborundum roughness elements of $0.8 \mathrm{~mm}$ diameter.

The boundary layer parameters were measured by planar PIV. The boundary layer thickness, $\delta_{99}$, equals approximately $11.9 \mathrm{~mm}$ at $x=-15 \mathrm{~mm}$ (Fig. 2). Other relevant boundarylayer parameters are summarized in Table 2, where the wall shear velocity was estimated by fitting with the law of the wall with $\kappa=0.41$ and $A_{2}=5.0$ following Pröbsting et al. (2016). The values are in good agreement with those reported by Pröbsting et al. (2015) in a similar experiment where measurements were performed using high-repetitionrate tomographic PIV. The results from the latter study are used for comparison to those obtained by the present lowrepetition-rate experiment.

Table 2 Turbulent boundary layer properties at $x=-15 \mathrm{~mm}$

\begin{tabular}{lll}
\hline Free-stream velocity & $u_{\infty}$ & $10 \mathrm{~m} / \mathrm{s}$ \\
Wall shear velocity & $u_{\tau}$ & $0.44 \mathrm{~m} / \mathrm{s}$ \\
Law-of-the-wall parameters & $\kappa$ & 0.41 \\
& $A_{2}$ & 5 \\
Boundary layer thickness & $\delta_{99}$ & $11.9 \mathrm{~mm}$ \\
Displacement thickness & $\delta^{*}$ & $2.2 \mathrm{~mm}$ \\
Reynolds number & $\operatorname{Re}_{\delta 99}$ & 8050 \\
& $\operatorname{Re}_{\delta}{ }^{*}$ & 1488 \\
& $\operatorname{Re}_{\tau}$ & 350 \\
\hline
\end{tabular}




\subsection{Low-repetition-rate tomographic PIV measurements}

The tomographic imaging system consists of four 16 MP LaVision Imager LX cameras equipped with Nikon $105 \mathrm{~mm}$ prime lenses set at an aperture of $f \#=16$. Scheimpflug adapters are used to adjust the object plane to be parallel to the wall and focused in the middle of the measurement volume. A dual-pulse Quantel Evergreen 200 laser (200 mJ pulse energy) is operated at $0.4 \mathrm{~Hz}$ with a $30 \mu$ s pulse separation. Fog droplets with $1 \mu \mathrm{m}$ diameter are used as tracer particles. The image source density of the recorded images equals 0.04 particles per pixel (ppp). The measurement volume, shaded green in Fig. 2, captures $30 \mathrm{~mm}$ in streamwise direction, $10 \mathrm{~mm}$ in spanwise direction and $5.6 \mathrm{~mm}$ in wall-normal direction, which corresponds to 50 percent of the boundary layer thickness. The average magnification equals $M=0.38$ and maximum particle displacement equals 15 voxels. A summary of the relevant experiment parameters is given in Table 3 .

A series of 50 particle image pairs is reconstructed using the MART technique (Elsinga et al. 2006). Crosscorrelation is done using a multi-pass volume deformation (Scarano and Riethmuller 2000) algorithm with final interrogation volume size equal to $40 \times 40 \times 40$ voxels $\left(0.78 \times 0.78 \times 0.78 \mathrm{~mm}^{3}\right)$ and with $75 \%$ overlap. The resulting vector spacing equals $0.19 \mathrm{~mm}$. Universal outlier detection (Westerweel and Scarano 2005) is applied to the vector fields to remove spurious vectors. The origin of the axis system is positioned at the plate-trailing edge (Fig. 2).

Table 3 Tomographic PIV experiment configuration

\begin{tabular}{ll}
\hline Seeding & $1 \mu \mathrm{m}$ fog droplets \\
Illumination & $\begin{array}{l}\text { Quantel Evergreen 200 Nd:YAG laser } \\
\text { Pulse energy 200 mJ at } 0.4 \mathrm{~Hz}\end{array}$ \\
Recording device & $\begin{array}{l}4 \times \text { LaVision Imager LX CCD camera } \\
\end{array}$ \\
Imaging & Nikon Micro-Nikkor objectives $f=105 \mathrm{~mm}$, \\
& $f \#=16$ \\
Pulse separation & $30 \mu \mathrm{s}$ \\
Acquisition frequency & $0.4 \mathrm{~Hz}$ \\
Measurement volume & $L_{x} \times L_{y} \times L_{z}=30 \times 10 \times 5.6 \mathrm{~mm}^{3}$ \\
& $L_{x} / \delta_{99} \times L_{y} / \delta_{99} \times L_{z} / \delta_{99}=2.5 \times 0.84 \times 0.47$ \\
& $L_{x}^{+} \times L_{y}^{+} \times L_{z}^{+}=900 \times 300 \times 170$ \\
Interrogation volume & $40 \times 40 \times 40 \mathrm{voxels}$ \\
& $0.78 \times 0.78 \times 0.78 \mathrm{~mm}^{3}$ \\
& $0.07 \times 0.07 \times 0.07 \mathrm{~mm}^{3} / \delta_{99}^{3}$ \\
& $23 \times 23 \times 23 v^{3} / u_{\tau}^{3}$ \\
Window overlap & $75 \%$ overlap \\
Grid size & $N_{x} \times N_{y} \times N_{z}=154 \times 52 \times 29$ vectors \\
\hline
\end{tabular}

\subsection{Microphone surface pressure measurements}

A Sonion 8010T microphone is mounted below a pinhole positioned $30 \mathrm{~mm}$ upstream of the trailing edge (red dot in Fig. 2), to measure surface pressure fluctuations to be compared with those extracted from tomographic PIV. Figure 3 shows a schematic of the pinhole-microphone arrangement. The microphone has a flat frequency response in the range of $0.25-11.5 \mathrm{kHz}$ and the pinhole arrangement is designed such that the resonance frequency of the cavity is beyond this range (i.e., $12 \mathrm{kHz}$, Alster 1972). The microphone signal is recorded over $90 \mathrm{~s}$ at $51.2 \mathrm{kHz}$ using an NI 9234 signal acquisition module that has a built-in anti-aliasing module. The microphone measurements are carried out after the PIV measurements. A frequency-dependent calibration (Zuckerwar et al. 2006) was carried out using a LinearX M53 reference microphone, which was calibrated by means of a GRAS 42AA piston phone. No further signal processing is applied. Spectral analysis is performed using a periodogram method with Hamming windows of $80 \mathrm{~ms}$, resulting in a frequency sampling of $12.5 \mathrm{~Hz}$.

\section{Results and discussion}

Two consecutive low-repetition-rate tomographic PIV velocity measurements are plotted in Fig. 4. The figure shows isosurfaces of high- and low-speed streaks (blue, $u-\bar{u}=u^{\prime}=-1.25 \mathrm{~m} / \mathrm{s}$; green, $u^{\prime}=+1.25 \mathrm{~m} / \mathrm{s}$ ) and vortices identified by the Q-Criterion (orange, $Q=10^{7} / \mathrm{s}^{2}$ ). The free-stream flow is directed along the $x$-axis. A typical turbulent boundary layer flow organization can be seen, with vortices positioned on top of the low-speed streaks. Two vortical structures, S1 and S2, are indicated in the figure by black arrows. At the measurement rate of $0.4 \mathrm{~Hz}$, there is $2.5 \mathrm{~s}$ in between the two consecutive velocity measurements. Taking the free-stream velocity as convective speed, the vortices are expected to travel approximately $25 \mathrm{~m}$ in this time corresponding to 400 measurement volume lengths. The actual convective speed in the turbulent boundary layer



Fig. 3 Pinhole microphone configuration for reference pressure power spectrum measurement 



Fig. 4 Two consecutive tomographic PIV measurements (left: $t=0 \mathrm{~ms}$, right: $t=2500 \mathrm{~ms}$ ). Isosurfaces show low- and high-speed streaks (blue, $u^{\prime}=-1.25 \mathrm{~m} / \mathrm{s}$; green, $u^{\prime}=+1.25 \mathrm{~m} / \mathrm{s}$ ) and vortices identified by the Q-Criterion (orange, $\mathrm{Q}=10^{7} / \mathrm{s}^{2}$ )

is expected to be lower, however. Taking the friction velocity as convective speed, structures are expected to travel over approximately $1.1 \mathrm{~m}$, corresponding to 30 measurement volume lengths. Therefore, at $t=2500 \mathrm{~ms}$, the structures $\mathrm{S} 1$ and $\mathrm{S} 2$ are not present in the measurement volume anymore.

\subsection{Finite time marching}

The measurement at time $t=0 \mathrm{~ms}$ (Fig. 4-left) is animated using both Taylor's hypothesis and the vorticity equation. For the VIC-based procedure, a time step of $10 \mu \mathrm{s}$ is required (Schneiders et al. 2014). This corresponds to a measurement frequency of $100 \mathrm{kHz}$. However, based on the interrogation volume size and free-stream velocity, the expected maximum resolved frequency (Eq. 9) of velocity fluctuations equals $6.4 \mathrm{kHz}$. Despite the small integration time step, it is, therefore, not expected that fluctuations beyond $6.4 \mathrm{kHz}$ are resolved. To reduce memory requirements, the results are stored at a reduced sampling rate of $33 \mathrm{kHz}$, which is still well beyond the expected maximum resolved frequency. Time marching is performed over $1.5 \mathrm{~ms}$, which equals half $T_{\max }$ (Eq. 6). The minimum resolved frequency equals accordingly approximately $0.667 \mathrm{kHz}$ (Eq. 7). Note that, the VIC-based procedure inherently applies a divergencefree filter to the measurement data. The recent literature has shown that this allows for important regularization of the measurement data (de Silva et al. 2013; Schiavazzi et al. 2014; Wang et al. 2016a, b). Therefore, the same is applied before using the Taylor's hypothesis to allow direct comparison of the time-marching techniques.

The computational time of the VIC-based approach is on the order of the computational time required for processing of a similar time-resolved measurement dataset. This is approximately an order of magnitude larger than the computational cost of application of Taylor hypothesis. The computational cost of the divergence-free filter is negligible (order of seconds per snapshot) in comparison to the other processing steps in the measurement chain.

Figure 5 shows perspective views of the resulting flow fields at $t=0.75 \mathrm{~ms}$ and side-views of the flow at $t=0,0.75$ and $1.5 \mathrm{~ms}$ (from top to bottom). The left figure shows the result from Taylor's hypothesis and the right figure the finite time vorticity marching result using VIC. The isosurfaces of vortices identified by the Q-Criterion are colored by spanwise distance for clarity of the side-view visualizations. The supplementary material contains video-animations of the full temporal velocity evolution in the time-segment.

The structures S1 and S2 are indicated in the perspective view, and have marched downstream for both the Taylor's hypothesis and VIC results. The side-view visualizations of both results show an empty region without vortical structures in the upstream flow region for $t>0$, as indicated by the dashed line. This eroded flow region is growing with time marching because the finite time marching techniques only leverage the measured flow information and do not provide a boundary condition for new structures entering into the domain, as discussed in Sect. 2.

A closer inspection to the perspective view at $t=0.75 \mathrm{~ms}$ shows less vortical structures in the near-wall region for the VIC result in comparison to the result from Taylor's hypothesis. The side views indicate that increased coherence of the vortical structures is obtained by the VIC-based result. In addition, the VIC-based approach employs side-padding of the measurement volume (Fig. 1), which results in marginally increased coherence of the vortical structures on the domain boundaries as visible in Fig. 5. The video-animations provided as supplementary material further illustrate the development of the near-wall vortical structures.

The coherence of velocity fluctuations is calculated and compared to the time-resolved tomographic PIV results 



Fig. 5 Isosurfaces of vortices identified by the Q-Criterion $\left(Q=10^{7}\right.$ / $\mathrm{s}^{2}$, colored by $z$ ) and low-speed streaks $\left(u^{\prime}=-1.25 \mathrm{~m} / \mathrm{s}\right.$, blue) as obtained using Taylor's hypothesis (left) and VIC (right). The per-

spective view is taken at $t=0.75 \mathrm{~ms}$. The side views show from top to bottom $t=0,0.75$ and $1.5 \mathrm{~ms}$. The dashed black line indicates the eroded area

obtained by Pröbsting et al. (2015). The results in the domain center along the full spanwise extent of the measurement volume are taken to increase convergence of the coherence estimation. The results are evaluated at the same wall-normal location $(y=3 \mathrm{~mm})$ as was used in Pröbsting et al. (2015). Note that, by Pröbsting et al. (2015), the highrepetition-rate results were evaluated $30 \mathrm{~mm}$ downstream at the location of the trailing edge (Fig. 2). In Fig. 6, the spanwise coherence of wall-normal velocity fluctuations at $2 \mathrm{kHz}$ (left) and $5 \mathrm{kHz}$ (right) is plotted. Pröbsting et al. (2015) found the spatial resolution of time-resolved tomographic PIV (window size indicated in Fig. 6) to be sufficient to capture fluctuations at $2 \mathrm{kHz}$, providing a reference for the current results.

The results from the low-repetition-rate system (blue and red lines) are found to be in good correspondence with the TR-PIV results. At the higher frequency of $5 \mathrm{kHz}$ (right figure), on the Nyquist limit of the time-resolved system, Pröbsting et al. (2015) reported that the coherence is overestimated due to spatial filtering. Because the

low-repetition-rate system uses a smaller correlation volume, a sharper coherence function than obtained by the time-resolved system is expected. This is confirmed by the results in Fig. 6-right. The result from Taylor's hypothesis and VIC-based finite time marching yields similar coherence, with the VIC-based result being marginally higher. Both techniques yield a coherence of 0.2 at approximately $25 \%$ smaller distance than the high-repetition-rate result.

\subsection{Pressure time evolution from a snapshot}

Pressure is calculated from the velocity temporal evolution in the time segments. Figure 7 shows the resulting isosurfaces of pressure (colored by $z, p=-0.5 \mathrm{~Pa}$ ) in a visualization similar to Fig. 6. In both the TH (left) and VIC (right) results, low-pressure regions can be seen to coincide with the vortical structures S1 and S2 highlighted earlier. First comparison of the $\mathrm{TH}$ and VIC results shows that the latter yields a smoother pressure field. In addition, the VIC-based result shows a large low-pressure region near the domain 



Fig. 6 Spanwise coherence of wall-normal velocity fluctuations at wall-normal height $y=3 \mathrm{~mm}$ at $2 \mathrm{kHz}$ (left) and $5 \mathrm{kHz}$ (right). Time-resolved PIV results reproduced from Pröbsting et al. (2015)
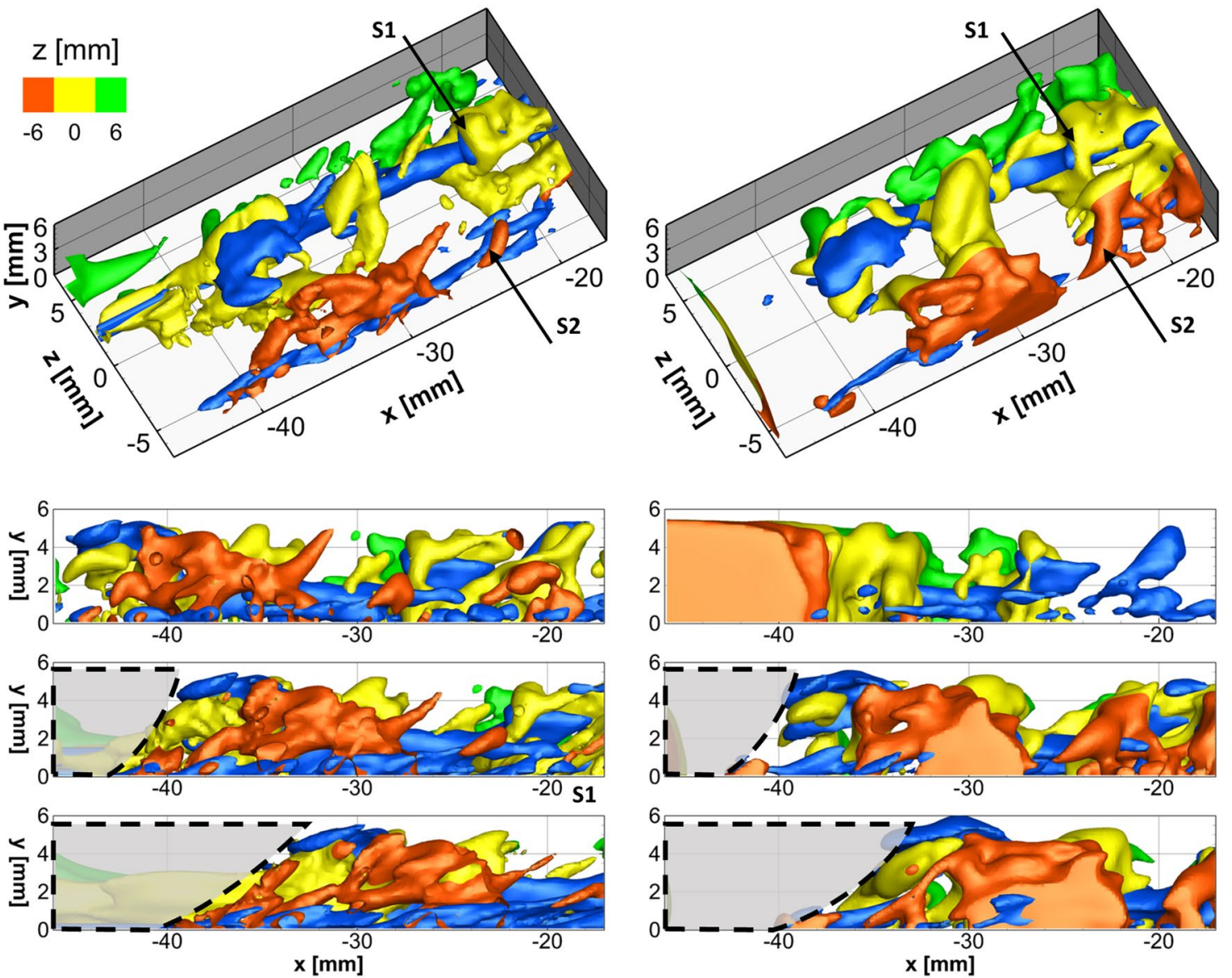

Fig. 7 Isosurfaces of low-pressure regions ( $p=-0.5 \mathrm{~Pa}$, colored by $z$ ) and low-speed streaks $\left(u^{\prime}=-1.25 \mathrm{~m} / \mathrm{s}\right.$, blue) as obtained using Taylor's hypothesis (left) and VIC (right). The perspective view is taken

at $t=0.75 \mathrm{~ms}$. The side-views show from top to bottom $t=0,0.75$ and $1.5 \mathrm{~ms}$. The dashed black line indicates the eroded area

The results in Fig. 7 show instantaneous pressure fields inflow, which disappears after a short time marching (see video animation in the supplementary material). at the measurement time-instant $\left(t_{0}=0\right)$ and the results obtained from the time-marching approaches at two later 
time instants ( $t=0.75 \mathrm{~ms}$ and $t=1.5 \mathrm{~ms})$. The latter two correspond to time-marching over $\Delta T=t_{0-} t=0.75$ and $1.5 \mathrm{~ms}$. The root mean squared (rms) surface pressure fluctuations can be evaluated at each $\Delta T$ by considering the data ensemble of all low-repetition-rate measurements. Inspection of the rms surface pressure fluctuations calculated at each time instant at the wall $(y=0)$ shows that the VIC-based result settles to a constant rms level of $0.6 \mathrm{~Pa}$ after $0.1 \mathrm{~ms}$ time marching (Fig. 8). This level compares well to the rms pressure fluctuation level obtained from the microphone measurements, that were band-pass filtered in the region where they return a flat response $(250-11 \mathrm{kHz})$. This compares well to the rms pressure level of $0.57 \mathrm{~Pa}$ that was measured in a similar turbulent boundary layer by Ghaemi et al. (2012). In

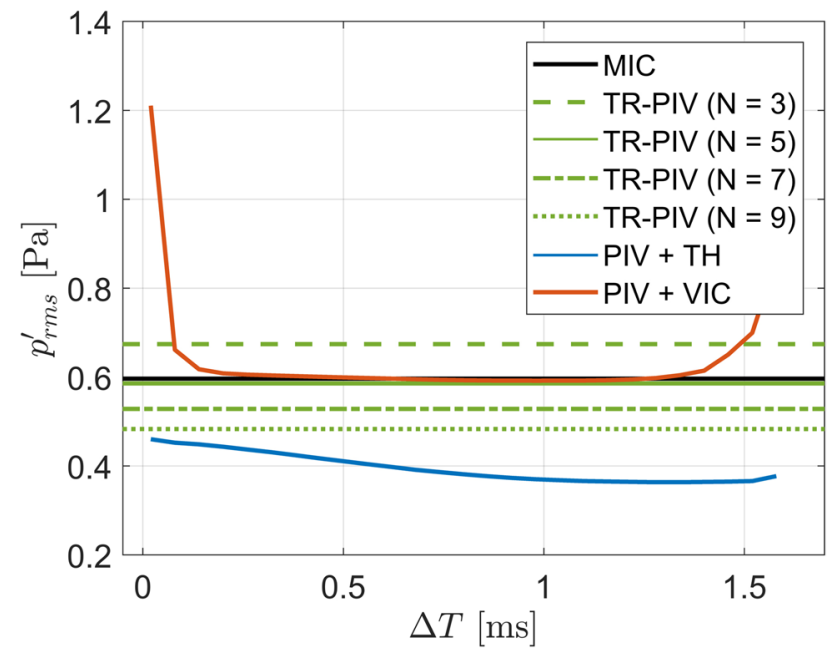

Fig. 8 Root mean squared surface pressure fluctuations obtained by the Taylor's hypothesis (blue line) and VIC-based (red line) approaches, calculated at each time instant of the time-marching simulation. For comparison, the reference microphone result (black line) and time-resolved PIV results by Pröbsting et al. (2015, green lines) are indicated in the figure addition, the time-resolved result by Pröbsting et al. (2015) using $N=5$ returns this rms value. The value $N$ indicates the number of consecutive snapshots used for evaluation of the velocity material derivative. Increasing this number corresponds to stronger filtering and thereby returns a lower rms level. The result obtained by Taylor's hypothesis yields the lowest rms pressure level (initially $0.46 \mathrm{~Pa}$ ), but does not suffer from the initial high rms peak value returned by the VIC analysis. In addition, at approximately $1.5 \mathrm{~ms}$, the VICbased result returns again an increased rms pressure value.

\subsection{Pressure coherence}

The magnitude of the spanwise coherence of surface $(y=0)$ pressure fluctuations is plotted in Fig. 9. This represents the cross-power spectral density of pressure fluctuations in two points at a spanwise separation $\Delta z$. Again, for calculation of the coherence, the results in the domain center along the full spanwise extent of the measurement volume are taken to increase convergence of the coherence estimation. In addition, again the high-repetition-rate results (Pröbsting et al. 2015) were evaluated $30 \mathrm{~mm}$ downstream at the location of the trailing edge (Fig. 2). The time-resolved PIV result (left figure) shows data up to a frequency of $5000 \mathrm{~Hz}$, which equals half the sampling frequency. The coherence is found to be highest in the low-frequency range and decreasing for increased frequencies. The low-repetition-rate results (middle and right figures) provide data up to higher frequencies, but lack the low-frequency range (dashed in the figures) due to the limited time segments that can be obtained from instantaneous velocity snapshots (Sect. 3.1). The results are similar to those obtained by the time-resolved approach, but a significantly smaller coherence is found in the high frequency range.

The lower coherence at high frequency is attributed to the smaller interrogation volume size that is achieved using the low-repetition-rate measurement system. To investigate the
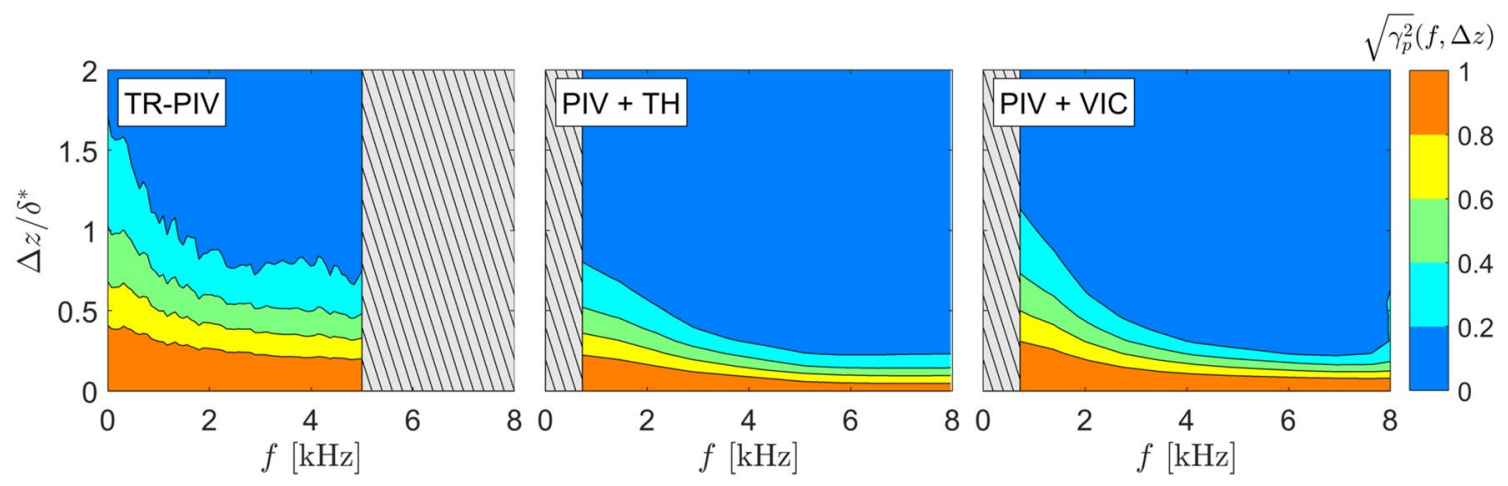

Fig. 9 Magnitude of the spanwise coherence of surface $(y=0)$ pressure fluctuations as obtained from time-resolved tomographic PIV by Pröbsting et al. (2015, left figure) and low-repetition-rate PIV using Taylor's hypothesis (middle) and VIC (right) 
effect of the interrogation volume size on the results, the data is also processed using a 50 and $100 \%$ larger interrogation volume size. In Fig. 10 the resulting coherence obtained using the VIC-based approach is given. Taking a threshold of 0.2 as the coherence length, a minimum coherence length is found at approximately $l_{\min }=3 \Delta z$, corresponding to $75 \%$ of the interrogation volume size. This is indicated by the dashed red line in the figure. The vertical dashed black line indicates the corresponding expected maximum resolved frequency calculated from Eq. (8). As expected, the minimum coherence length and the maximum resolved frequency increases when interrogation volume size is reduced. When the interrogation volume size is reduced to $0.8 \mathrm{~mm}$, noise is found to start dominating the high-frequency result, indicating that the interrogation volume size cannot be reduced further. The latter interrogation volume size is used in the remainder of this work.

Pröbsting et al. (2015) computed the correlation length from the results by means of an exponential fit. These results are reproduced in Fig. 11. The results from the models by Corcos (1964) and Efimtsov (1982) that were used for comparison by Pröbsting et al. (2015) are also reproduced in the figure. The result from high-repetition-rate PIV is found to correspond well to the models around $1 \mathrm{kHz}$, but flattens for higher frequencies.

In the high-frequency range, the low-repetition-rate results using both Taylor's hypothesis and VIC show good correspondence with the Corcos and Efimtsov models, as expected from the fact that these methods benefit from increased spatial resolution. The approach using Taylor's hypothesis shows a lower coherence length than the VIC result for frequencies below $2 \mathrm{kHz}$. The VIC result follows the Efimtsov model and returns the same coherence length as high-repetition-rate PIV in the range of $0.7-1 \mathrm{kHz}$. Reduced accuracy of the result of Taylor's hypothesis at low frequencies could be expected, as the assumption of frozen turbulence is not valid for large structures corresponding to low frequencies. The non-linear VIC-based model appears

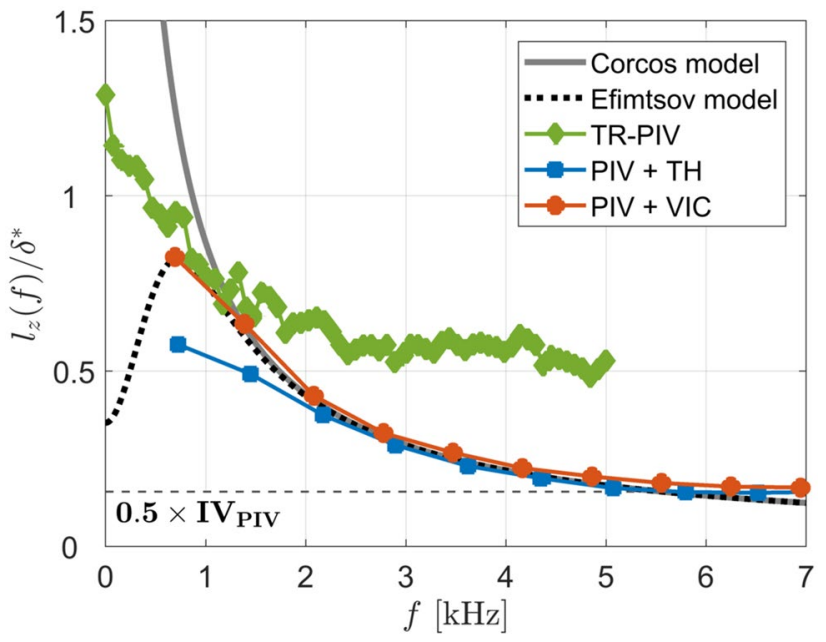

Fig. 11 Spanwise coherence length of surface $(y=0)$ pressure fluctuations

to improve upon this, as also observed by Schneiders et al. (2014).

\subsection{Pressure spectrum}

The pressure spectrum calculated from the microphone measurements is represented with a gray line in Fig. 12. The resonance frequency of the pinhole microphone system was estimated at $12 \mathrm{kHz}$ (Sect. 3.2), which is close to the peak region in the present experiments. The high-repetitionrate tomographic PIV experiments performed by Pröbsting et al. (2015) at a measurement rate of $10 \mathrm{kHz}$ yielded pressure fluctuations up to $5 \mathrm{kHz}$. These are shown in Fig. 8 by the blue markers. Note that, the high-repetition-rate results were evaluated at the trailing edge location, whereas the microphone location is $30 \mathrm{~mm}$ upstream of the trailing edge (Fig. 2).

The high-repetition-rate results showed a significant dependence upon the length of the temporal kernel used


Fig. 10 Magnitude of the spanwise coherence of surface $(y=0)$ pressure fluctuations as obtained from low-repetition-rate PIV with VIC timemarching at three different window sizes 
Fig. 12 Pressure power spectral density at $x=-30 \mathrm{~mm}$. The dashed lines indicate the expected minimum and maximum resolvable frequencies with finite time-marching analysis. The time-resolved PIV data are taken at $x=0 \mathrm{~mm}$ and reproduced from a previous study in a similar flow configuration (Pröbsting et al. 2015)



to estimate the material derivative $(N=3,5,7$ and 9 snapshots). The pressure spectrum estimated with a relatively short kernel overestimates the pressure fluctuation amplitude in the high-frequency range, which is ascribed to the effect of measurement noise. The latter is plausible causing the noise floors evident at higher frequencies. A longer kernel does filter out the noise but at the cost of damping part of the fluctuations due to physical phenomena. This is particularly evident for $N=9$. With a kernel of $N=5$ or 7 time-resolved tomographic PIV results follow the reference microphone data reasonably well up to a frequency of $3.5 \mathrm{kHz}$. Reliable results are, therefore, obtained up to a frequency $30 \%$ below the Nyquist frequency $(5 \mathrm{kHz})$, which is ascribed to the limited spatial resolution of the high-repetition-rate PIV measurement system. The work of Pröbsting et al. (2015) concluded that the results using kernel sizes $N=5$ and 7 are most accurate. Accordingly, these should be considered most accurate for comparison with the current low-repetition-rate approaches.

The pressure spectrum is also evaluated at the microphone location ( $x=-30 \mathrm{~mm}$, Fig. 2) for the approaches using low-repetition-rate PIV. As expected from the discussion on the coherence length, the Taylor's hypothesis (blue line) approach shows acceptable results in the high-frequency range. In the low-frequency range, an underestimation of the pressure is found. The VIC-based result follows the microphone measurements with similar accuracy as the high-repetition-rate tomographic PIV data with kernel sizes $N=5$ and 7 . Beyond $3.5 \mathrm{kHz}$, the VIC result maintains a good agreement with the microphone data. Based on Eq. (9) the maximum resolvable frequency was estimated around $6.4 \mathrm{kHz}$. A departure of the microphone results is found at approximately $5 \mathrm{kHz}$. This is attributed to the convective velocity of the pressure fluctuations being typically lower than the free-stream velocity (Panton and Linebarger 1974).

A more quantitative inspection of the results is possible by considering the difference between the reference spectrum obtained from the transducer and the PIV results. In Fig. 13 this difference is plotted for the relevant range of frequencies. As expected from the discussion above, the highrepetition-rate tomographic PIV result shows a noise floor starting from approximately $3 \mathrm{kHz}$. The smallest resolved power equals $10^{-6} \mathrm{~Pa}^{2} / \mathrm{Hz}$, as indicated by the dotted line in Fig. 13. The Taylor's hypothesis and VIC-based results that use low-repetition rate measurement results show a more than an order of magnitude smaller noise floor at $5 \times 10^{-8}$ $\mathrm{Pa}^{2} / \mathrm{Hz}$. In the lower frequency range $(1-2 \mathrm{kHz})$ the highrepetition rate and low-repetition rate VIC results show similar error levels on the order of $20 \%$ of the reference. As expected from the discussion above, the Taylor's hypothesis results shows increased errors of around $50 \%$ of the reference.

\section{Conclusions}

The work shows that the power spectral density and coherence of temporal pressure fluctuations can be obtained from low-repetition-rate tomographic PIV measurements. Theoretical analysis shows that spatial resolution (i.e., interrogation volume size) is inversely proportional to the maximum resolved frequency. Higher spatial resolution of low-repetition-rate measurements in comparison to high-repetition-rate measurements is, therefore, expected to allow for a linear increase in maximum resolved frequency. Spectral information is obtained from the low-repetition-rate measurements 
Fig. 13 Difference between the reference pressure spectrum obtained from the microphone measurements and the PIV results. The time-resolved PIV data is reproduced from a previous study in a similar flow configuration (Pröbsting et al. 2015)



by finite time marching using Taylor's hypothesis or a VICbased approach. The experimental assessment shows that both the approach based on Taylor's hypothesis and VIC allow for acceptable results in the high-frequency range. The noise floor of the high-repetition-rate result is found at $10^{-6} \mathrm{~Pa}^{2} / \mathrm{Hz}$. Instead, the low-repetition-rate techniques show a lower noise floor at $5 \times 10^{-8} \mathrm{~Pa}^{2} / \mathrm{Hz}$. For lower frequencies (below $2 \mathrm{kHz}$ ) corresponding to larger structures, the assumption of frozen turbulence does not hold and an underestimation of the pressure fluctuations is found by the results based on Taylor's hypothesis (on the order of 50\% error). The VIC-based approach is able to obtain spectral information in this range with similar accuracy as a highrepetition-rate measurement system ( $20 \%$ error).

Acknowledgements The research is partly funded by LaVision $\mathrm{GmbH}$.

Open Access This article is distributed under the terms of the Creative Commons Attribution 4.0 International License (http://creativeco mmons.org/licenses/by/4.0/), which permits unrestricted use, distribution, and reproduction in any medium, provided you give appropriate credit to the original author(s) and the source, provide a link to the Creative Commons license, and indicate if changes were made.

\section{References}

Adrian RJ (1997) Dynamic ranges of velocity and spatial resolution of particle image velocimetry. Meas Sci Technol 8:1393. https ://doi.org/10.1088/0957-0233/8/12/003

Adrian RJ, Westerweel J (2011) Particle image velocimetry. Cambridge University Press, Cambridge

Alster M (1972) Improved calculation of resonant frequencies of Helmholtz resonators. J Sound Vib 24:63-85. https://doi. org/10.1016/0022-460X(72)90123-X
Baur T, Köngeter J (1999) PIV with high temporal resolution for the determination of local pressure reductions from coherent turbulence phenomena. In: 3rd Int. Work PIV, Santa Barbara

Bitter M, Hara T, Hain R, Yorita D, Asai K, Kähler CJ (2012) Characterization of pressure dynamics in an axisymmetric separating/reattaching flow using fast-responding pressure-sensitive paint. Exp Fluids 53:1737. https://doi.org/10.1007/s0034 8-012-1380-7

Charonko JJ, King CV, Smith BL, Vlachos PP (2010) Assessment of pressure field calculations from particle image velocimetry measurements. Meas Sci Technol 21:105401. https://doi. org/10.1088/0957-0233/21/10/105401

Christiansen JP (1973) Numerical simulation of hydrodynamics by the method of point vortices. J Comput Phys 13:363-379. https://doi. org/10.1016/0021-9991(73)90042-9

Corcos GM (1964) The structure of the turbulent pressure field in boundary-layer flows. J Fluid Mech 18:353-378. https://doi. org/10.1017/S002211206400026X

Cuzol A, Mémin E (2009) A stochastic filtering technique for fluid flow velocity fields tracking. IEEE Trans Pattern Anal Mach Intell 31:1278-1293. https://doi.org/10.1109/TPAMI.2008.152

de Kat R, Ganapathisubramani B (2012) Pressure from particle image velocimetry for convective flows: a Taylor's hypothesis approach. Meas Sci Technol 24:024002. https://doi.org/10.1088/09570233/24/2/024002

de Kat R, van Oudheusden BW (2012) Instantaneous planar pressure determination from PIV in turbulent flow. Exp Fluids 52:10891106. https://doi.org/10.1007/s00348-011-1237-5

de Silva MC, Philip J, Marusic I (2013) Minimization of divergence error in volumetric velocity measurements and implications for turbulence statistics. Exp Fluids 54:1557. https://doi.org/10.1007/ s00348-013-1557-8

Dong P, Hsu TY, Atsavaprenee P, Wei T (2001) Digital particle accelerometry. Exp Fluids 30:626-632. https://doi.org/10.1007/s0034 80000240

Efimtsov BM (1982) Characteristics of the field of turbulent wall pressure fluctuations at large Reynolds numbers. Sov Phys Acoust 28:289-292

Elsinga GE, Scarano F, Wieneke B, van Oudheusden BW (2006) Tomographic particle image velocimetry. Exp Fluids 41:933-947. https://doi.org/10.1007/s00348-006-0212-z 
Ferrari S, Rossi L (2008) Particle tracking velocimetry and accelerometry (PTVA) measurements applied to quasi-two-dimensional multi-scale flows. Exp Fluids 44:873-886. https://doi.org/10.1007/ s00348-007-0443-7

Fujisawa N, Tanahashi S, Srinavas K (2005) Evaluation of pressure field and fluid forces on a circular cylinder with and without rotational oscillation using velocity data from PIV measurement. Meas Sci Technol 16:989-996. https://doi.org/10.1088/0957-0233/16/4/011

Gesemann S, Huhn F, Schanz D, Schröder A (2016) From noisy particle tracks to velocity, acceleration and pressure fields using B-splines and penalties. In: 18th Int. Symp. on Applications of Laser and Imaging Techniques to Fluid Mechanics. Lisbon, Portugal, 4-7 July 2016

Ghaemi S, Ragni D, Scarano F (2012) PIV-based pressure fluctuations in the turbulent boundary layer. Exp Fluids 53:1823-1840. https ://doi.org/10.1007/s00348-012-1391-4

Gurka R, Liberzon A, Hefetz D, Rubinstein D, Shavit U (1999) Computation of pressure distribution using PIV velocity data. In: 3rd Int. Workshop on Particle Image Velocimetry, Santa Barbara, pp 671-676

Haigermoser C (2009) Application of an acoustic analogy to PIV data from rectangular cavity flows. Exp Fluids 47:145-157. https://doi. org/10.1007/s00348-009-0642-5

Hain R, Kähler CJ, Michaelis D (2008) Tomographic and time resolved PIV measurements on a finite cylinder on a flat plate. Exp Fluids 45:715-724. https://doi.org/10.1007/s00348-008-0553-x

Hosokawa S, Moriyama S, Tomiyama A, Takada N (2003) PIV measurement of pressure distributions about single bubbles. J Nucl Sci Technol 40:754-762. https://doi.org/10.1080/18811 248.2003.9715416

Huhn F, Schanz D, Gesemann S, Schröder A (2016) FFT integration of instantaneous 3D pressure gradient fields measured by Lagrangian particle tracking in turbulent flows. Exp Fluids 57:151. https://doi. org/10.1007/s00348-016-2236-3

Imaichi K, Ohmi K (1983) Numerical processing of flow-visualization pictures-measurement of two-dimensional flow. J Fluid Mech 129:283-311. https://doi.org/10.1017/S0022112083000774

Jensen A, Pedersen GK (2004) Optimization of acceleration measurements using PIV. Meas Sci Technol 15:2275-2283. https://doi. org/10.1088/0957-0233/15/11/013

Jeon YJ, Chatellier L, David L (2014) Fluid trajectory evaluation based on an ensemble-averaged cross-correlation in time-resolved PIV. Exp Fluids 55:1766. https://doi.org/10.1007/s00348-014-1766-9

Joshi P, Liu X, Katz J (2012) Modification of turbulence in boundary layers by mean and fluctuating pressure gradients FEDSM201272280: ASME 2012 Fluids Engineering Summer Meeting, Puerto Rico

Kotsonis M, Ghaemi S, Veldhuis L, Scarano F (2011) Measurement of the body force field of plasma actuators. J Phys D Appl Phys 44:045204. https://doi.org/10.1088/0022-3727/44/4/045204

Kurtulus DF, Scarano F, David L (2007) Unsteady aerodynamic forces estimation on a square cylinder by TR-PIV. Exp Fluids 42:185196. https://doi.org/10.1007/s00348-006-0228-4

Laskari A, de Kat R, Ganapathisubramani B (2016) Full-field pressure from snapshot and time-resolved volumetric PIV. Exp Fluids 57:44. https://doi.org/10.1007/s00348-016-2129-5

Lemke M, Sesterhenn J (2016) Adjoint-based pressure determination from PIV data in compressible flows-validation and assessment based on synthetic data. Eur J Mech B/Fluids 58:29-38. https:// doi.org/10.1016/j.euromechflu.2016.03.006

Liu X, Katz J (2006) Instantaneous pressure and material acceleration measurements using a four-exposure PIV system. Exp Fluids 41:227. https://doi.org/10.1007/s00348-006-0152-7

Lynch K, Scarano F (2013) A high-order time-accurate interrogation method for time-resolved PIV. Meas Sci Technol 24:035305. https ://doi.org/10.1088/0957-0233/24/3/035305
Lynch KP, Scarano F (2014) Material acceleration estimation by four-pulse tomo-PIV. Meas Sci Technol 25:084005. https://doi. org/10.1088/0957-0233/25/8/084005

Lynch KP, Pröbsting S, Scarano F (2014) Temporal resolution of time-resolved tomographic PIV in turbulent boundary layers. In: Proceedings of the 17th international symposium on applications of laser techniques to fluid mechanics, Lisbon, Portugal, 7-10 July, 2014

McClure J, Yarusevych S (2017) Instantaneous PIV/PTV-based pressure gradient estimation: a framework for error analysis and correction. Exp Fluids 58:92. https://doi.org/10.1007/s0034

Neeteson NJ, Rival DE (2015) Pressure-field extraction on unstructured flow data using a Voronoi tessellation-based networking algorithm: a proof-of-principle study. Exp Fluids 56:44. https ://doi.org/10.1007/s00348-015-1911-0

Neeteson NJ, Bhattacharya S, Rival DE, Michaelis D, Schanz D, Schröder A (2016) Pressure-field extraction from Lagrangian flow measurements: first experiences with 4D-PTV data. Exp Fluids 57:102. https://doi.org/10.1007/s00348-016-2170-4

Novara M, Scarano F (2013) A particle-tracking approach for accurate material derivative measurements with tomographic PIV. Exp Fluids 54:1584. https://doi.org/10.1007/s00348-013-1584-5

Novara M, Schanz D, Reuther N, Kähler CJ, Schröder A (2016) Lagrangian 3D particle tracking in high-speed flows: shakethe-box for multi-pulse systems. Exp Fluids 57:128. https://doi. org/10.1007/s00348-016-2216-7

Panton RL, Linebarger JH (1974) Wall pressure spectra calculations for equilibrium boundary layers. J Fluid Mech 65:261-287. https://doi.org/10.1017/S0022112074001388

Perret L, Braud P, Fourment C, David L, Delville J (2006) 3-Component acceleration field measurement by dual-time stereoscopic particle image velocimetry. Exp Fluids 40:813-824. https://doi. org/10.1007/s00348-006-0121-1

Pröbsting S, Scarano F, Bernardini M, Pirozzoli S (2013) On the estimation of wall pressure coherence using time-resolved tomographic PIV. Exp Fluids 54:1567. https://doi.org/10.1007/s0034 8-013-1567-6

Pröbsting S, Tuinstra M, Scarano F (2015) Trailing edge noise estimation by tomographic particle image velocimetry. J Sound Vib 346:117-138. https://doi.org/10.1016/j.jsv.2015.02.018

Pröbsting S, Schneiders JFG, Avallone F, Ragni D, Scarano F (2016) Trailing-edge noise diagnostics with low-repetition-rate PIV. 22nd AIAA/CEAS Aeroacoustics Conference, Aeroacoustics Conferences, (AIAA 2016-3023). https://doi.org/10.2514/6.2016-3023

Ragni D, Ashok A, van Oudheusden BW, Scarano F (2009) Surface pressure and aerodynamic loads determination of a transonic airfoil based on particle image velocimetry. Meas Sci Technol 20:074005. https://doi.org/10.1088/0957-0233/20/7/074005

Ragni D, van Oudheusden BW, Scarano F (2012) 3D pressure imaging of a propeller blade tip region by phase-locked stereoscopic PIV. Exp Fluids 52:463-477

Scarano F, Moore P (2011) An advection-based model to increase the temporal resolution of PIV time series. Exp Fluids 52:919-933. https://doi.org/10.1007/s00348-011-1158-3

Scarano F, Riethmuller ML (2000) Advances in iterative multigrid PIV image processing. Exp Fluids 29:S051-S060. https://doi. org/10.1007/s003480070007

Schanz D, Schroder A, Heine B, Dierksheide U (2012) Flow structure identification in a high-resolution tomographic PIV data set of the flow behind a backward facing step. In: Proceedings of the 16th international symposium on applications of laser techniques to fluid mechanics, Lisbon, Portugal, 9-12 July 2012

Schanz D, Gesemann S, Schröder A (2016) Shake-The-Box: Lagrangian particle tracking at high particle image densities. Exp Fluids 57:70. https://doi.org/10.1007/s00348-016-2157-1 
Schiavazzi D, Coletti F, Iaccarino G, Eaton JK (2014) A matching pursuit approach to solenoidal filtering of three-dimensional velocity measurements. J Comput Phys 263:206-221. https:// doi.org/10.1016/j.jcp.2013.12.049

Schneiders JFG, Scarano F (2016) Dense velocity reconstruction from tomographic ptv with material derivatives. Exp Fluids 57:139. https://doi.org/10.1007/s00348-016-2225-6

Schneiders JFG, Dwight RP, Scarano F (2014) Time-supersampling of 3D-PIV measurements with vortex-in-cell simulation. Exp Fluids 55:1692. https://doi.org/10.1007/s00348-014-1692-x

Schneiders JFG, Pröbsting S, Dwight RP, van Oudheusden BW, Scarano F (2016) Pressure estimation from single-snapshot tomographic PIV in a turbulent boundary layer. Exp Fluids 57:53. https ://doi.org/10.1007/s00348-016-2133-9

Schneiders JFG, Scarano F, Elsinga G (2017) Resolving vorticity and dissipation in a turbulent boundary layer by tomographic PTV and VIC+. Exp Fluids 58:27. https://doi.org/10.1007/s0034 8-017-2318-x

Schrijer FFJ, Scarano F (2008) Effect of predictor-corrector filtering on the stability and spatial resolution of iterative PIV interrogation. Exp Fluids 45:927-941. https://doi.org/10.1007/s0034 8-008-0511-7

Schröder A, Herr M, Lauke T, Dierksheide U (2006) A study on trailing-edge noise sources using high-speed particle image velocimetry. Notes Numer Fluid Mech 92:373-380. https://doi. org/10.1007/978-3-540-33287-9_46

Suzuki T, Ji H, Yamamoto F (2009) Unsteady PTV velocity field past an airfoil solved with DNS: Part 1. Algorithm of hybrid simulation and hybrid velocity field at $\operatorname{Re} \approx 103$. Exp Fluids 47:957-976. https://doi.org/10.1007/s00348-009-0691-9

Tronchin T, David L, Farcy A (2015) Evaluation of pressure field and fluid forces for 3D flow around flapping wing. Exp Fluids 56:1. https://doi.org/10.1007/s00348-014-1870-x

van Oudheusden BW (2013) PIV-Based pressure measurement. Meas Sci Technol 24:032001. https://doi.org/10.1088/09570233/24/3/032001

van Oudheusden BW, Scarano F, Roosenboom EW, Casimiri EW, Souverein LJ (2007) Evaluation of integral forces and pressure fields from planar velocimetry data for incompressible and compressible flows. Exp Fluids 43:153. https://doi.org/10.1007/s0034 8-007-0261-y van Gent P, Michaelis D, van Oudheusden BW, Weiss P-É, de Kat R, Laskari A, Jeon YJ, David L, Schanz D, Huhn F, Gesemann S, Novara M, McPhaden C, Neeteson N, Rival D, Schneiders JFG, Schrijer F (2017) Comparative assessment of pressure field reconstructions from particle image velocimetry measurements and Lagrangian particle tracking. Exp Fluids 58:33. https://doi. org/10.1007/s00348-017-2324-Z

Violato D, Moore P, Scarano F (2011) Lagrangian and Eulerian pressure field evaluation of rod-airfoil flow from time-resolved tomographic PIV. Exp Fluids 50:1057-1070. https://doi.org/10.1007/ s00348-010-1011-0

Wang C, Gao Q, Wang H, Wei R, Li T, Wang J (2016a) Divergencefree smoothing for volumetric PIV data. Exp Fluids 57:15. https ://doi.org/10.1007/s00348-015-2097-1

Wang Z, Gao Q, Wang C, Wei R, Wang J (2016b) An irrotation correction on pressure gradient and orthogonal-path integration for PIV-based pressure reconstruction. Exp Fluids 57:104. https://doi. org/10.1007/s00348-016-2189-6

Wang Z, Gao Q, Pan C, Feng L, Wang J (2017a) Imaginary particle tracking accelerometry based on time-resolved velocity fields. Exp Fluids 58:113. https://doi.org/10.1007/s00348-017-2394-y

Wang CY, Gao Q, Wei RJ, Li T, Wang JJ (2017b) Spectral decomposition-based fast pressure integration algorithm. Exp Fluids 58:84. https://doi.org/10.1007/s00348-017-2368-0

Welch PD (1967) The use of fast Fourier transform for the estimation of power spectra: a method based on time averaging over short, modified periodograms. IEEE Trans Audio Electroacoust AU 15:70-73. https://doi.org/10.1109/TAU.1967.1161901

Westerweel J, Scarano F (2005) Universal outlier detection for PIV data. Exp Fluids 39:1096-1100. https://doi.org/10.1007/s0034 8-005-0016-6

Yegavian R, Leclaire B, Champagnat F, Marquet O (2015) Performance assessment of PIV super-resolution with adjoint-based data assimilation. In: Proceedings of the 11th Int. Symp. on PIV, Santa Barbara, CA, US, 14-16 Sep 2015

Zuckerwar AJ, Herring GC, Elbing BR (2006) Calibration of the pressure sensitivity of microphones by a free-field method at frequencies up to $80 \mathrm{kHz}$. J Acoust Soc Am 119:320. https://doi. org/10.1121/1.2141360 